in the process. On admission to hospital there was no further aggressive behaviour, and the sudden alteration in the patient's conduct could not be attributed to any ictal or post-ictal events.

It is probable that in this case diazepam could be incriminated in the precipitation of aggressive behaviour. Though there was a long history of epilepsy, there was no evidence of alteration in epileptic status clinically, nor could personality deterioration be demonstrated. Further, E.E.G. recordings had been made 15 and four years previously, and a recent record revealed no deterioration. The E.E.G.s consistently showed paroxysms of polyspike activity, there being no indication of temporal lobe foci. It is, of course, well recognized that temporal lobe epilepsy or a temporal lobe abnormality carries an increased risk of personality disorder or behaviour disturbance. $^{2}$

If drugs of the benzodiazepine group are occasionally responsible for the release of aggressive outbursts, then caution is necessary in their administration not only for anxiety and tension, but perhaps even more so in epileptic disorders, where diazepam in particular has gained some popularity on account of this combination of anxiolytic and anticonvulsant properties. Paradoxical reactions with excitement are already known to occur. ${ }^{3}-\mathrm{I}$ am, etc.,

E. B. GORDON

Saxondale Hospital,

Radcliffe-on-Trent, Notts

1 Ingram, I. M., and Timbury, G. D., Lancet, $1960,2,766$

Herrington, R. N., Current Problems in Neuropsychiatry, British fournal of Psychiatry Special Publication No. 4, 1969.

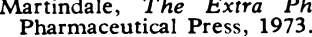

\section{Postoperative Management after} Thymectomy

SIR,-In their interesting paper on the management of thymectomy (8 February, p. 309) Dr. A. B. Loach and colleagues were unwise, in our view, to have proposed a critical level of 2 litres for the preoperative vital capacity with respect to the need for postoperative artificial ventilation. The number of patients in their study (21) is surely too small to justify such a conclusion. Indeed in their own series there was one exception, and of their eight other patients who required artificial ventilation no fewer than four had values for the vital capacity that lay so close to the "critical" level (one was actually 2 litres) that any confidence in it must be immediately undermined, given the variability inherent in the measurement. While the authors properly stress that other clinical factors must be taken into account when the vital capacity is close to the critical level, the concept of such a level is nevertheless likely to invite a false sense of security in those who choose to use it.

Dr. Loach and his colleagues suggest that there are two "safe" policies for management of the postoperative period-namely, either preoperative tracheostomy with artificial ventilation and withdrawal of anticholinesterases or (their own recommendation) an unchanged drug regimen in the postoperative period with tracheostomy selected principally on the preoperative vital capacity. We would like to propose an alternative which is in current use here. Nasotracheal intubation is undertaken at the time of induction of anaesthesia and anticholinesterase medication is substantially reduced or withdrawn for, like Osserman and Genkins, ${ }^{1}$ we have usually found the drug requirements to be reduced postoperatively. An edrophonium (Tensilon) test is carried out daily to assess anticholinesterase requirements and the vital capacity is charted four-hourly. The patient breathes spontaneously oxygenenriched air. If respiratory distress develops artificial ventilation is begun, and in some cases in which the vital capacity is low artificial ventilation is also instituted electively at night. After the fourth postoperative day a steadily increasing vital capacity is usually a sign that the tube may be removed. ${ }^{2}$

A potential drawback of our method is the discomfort associated with the presence of a nasotracheal tube, but in the event it has proved to be well tolerated. This management policy seems to us to retain the safeguard of a secure airway, which is lacking in the regimen proposed by Dr. Loach and his colleagues, while avoiding the morbidity associated with tracheostomy.-We are, etc.,

L. LOH J. Newsom Davis

National Hospital for Nervous Diseases,
London W.C.1

1 Osserman, K. E., and Genkins, G., Mount Sinai Fournal of Medicine, 1971, 38, 497. Newsom Davis, J., British fournal of Hospital
Medicine, 1974, 11, 933.

SIR,-We read the paper by Dr. A. B. Loach and others (8 February, p. 309) with interest and, while applauding the application of clinical measurement in patient management, we would like to make the following observations.

We agree that preoperative ventilatory function tests are of value in anticipating ventilatory support requirements in the postoperative period. However, the suggestion that a static vital capacity measuremen of less than 2 litres (irrespective of sex and weight) is the principal criterion for a safe management policy in which 12 days of artificial ventilation via a tracheostomy becomes mandatory appears to us to be far too rigid.

Undoubtedly the advent of the intensive care unit, assisted ventilation, and the closest co-operation between clinician and anaesthetist has now made surgery of the thymus gland for myasthenia a relatively safe pro cedure. While we agree with many of the issues raised in the article, there are certain different approaches which we consider worth mentioning. These are best highlighted by briefly indicating our routine.

A prolonged inpatient spell to prepare the patient, and staff, thoroughly for thymectomy is considered wise.

The administration of cholinesterase inhibitors is continued right up to the time of the operation.

We believe it good practice that every patient undergoing thymectomy should be nursed for at least 48 hours in the intensive care unit.

It is our experience that cholinesterase inhibitors are not necessary in the immediate postoperative period. Careful observation will usually indicate their need within 6-12 hours after thymeotomy. At such time the drugs may be restarted, usually in a reduced dosage.
Unless the patient already has a tracheostomy we find that this procedure is seldom necessary. But in patients who have poor pulmonary function (for example, a vital capacity of less than $1.5 \mathrm{l}$ ), when the pleura was inadvertently opened at the operation, or when there is any doubt about adequate ventilation, then we never hesitate to leave the endotracheal tube in situ and to ventilate the patient for at least 48 hours. Analgesics are given liberally, but no other drugs. After 48 hours the cholinergic drugs are restarted and usually within 24-48 hours the patient can be weaned off the ventilator and may then be extubated. Otherwise, with careful supervision, the patient may remain thus intubated for 5-7 days. We have found it to be exceptional for the patient then to need tracheostomy.

Each patient is a problem on his own and only careful and continuous observation by experienced personnel will point the way to the dosage and frequency of the drugs and the need for assisted ventilation. ${ }^{1-3}$ _We are, etc.,

M. J. LANGE

Royal Free Hospital,

T. Hilary Howells

London N.W.3

Lange, M. J., British fournal of Surgery, 1960, 48,285

Lange, M. J., Practitioner, 1966, 196, 448

of Medicine, J 1968, 61, 751. of the Royal Society

\section{Measurement of Neonatal Growth}

SIR,-The studies of Dr. H. B. Valman and his colleagues (3 August, p. 319) and Dr. Sheila A. McKenzie (4 January, p. 38) have again drawn attention to the uncertainty which surrounds the question of optimum milk requirements for low-birth-weight infants in the early weeks of life.

One reason for this continuing uncertainty is a persistence in using weight gain as the main standard of reference for neonatal growth. In clinical practice weight gain is still the most widely used measure of neonatal growth, but it should not be relied upon as the sole measure since it can so readily be influenced by factors which are not generally considered to be growth, such as variations in the state of hydration, fat, and mineral aocumulation. ${ }^{1}$ In older ahildren length or height is a most important measurement and in neonates it is a much better single criterion of growth than is weight since it is unaffected by the accumulation of water and fat. ${ }^{1}$ Head circumference is also an important measurement in the neonatal period since it accurately reflects intracranial volume ${ }^{2}$ and cellular brain growth. ${ }^{3}$ It is difficult therefore to understand why these other measures of growth are so infrequently used in studies of infant growth and nutrition.

The accompanying figures illustrate the importance of evaluating growth of length and head circumference in addition to weight gain. These show the growth during the first month of life of 23 healthy preterm infants who were born between 28 and 32 weeks' gestation. The infants were fed either expressed breast milk or a modified cow's milk. Average daily milk intake between the 7 th and 28 th days was $204 \mathrm{ml} / \mathrm{kg}$. The infants were weighed on an Avery scales accurate to $10 \mathrm{~g}$, crown-heel length was measured with a Neonatometer, ${ }^{4}$ and the 

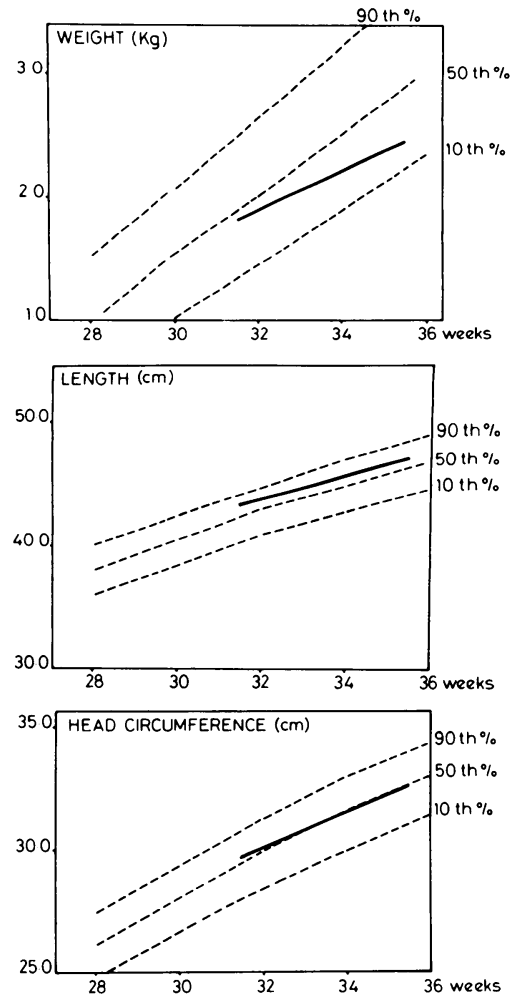

maximum occipitofrontal head circumference with a paper tape measure.

Growth in length and head circumference followed their expected intrauterine curves ${ }^{5}$ far more closely than did weight gain, which was markedly deflected from its expected course. If optimum postnatal growth in preterm infants is that which would have been attained in utero had gestation continued normally, ${ }^{6}$ then these infants might have been diagnosed as failing to grow adequately had weight been the only measure of growth used. However, the much closer approximation of head and linear growth to expected intrauterine patterns suggests that in fact the infants were growing very well. By using these other parameters a far better impression of overall body growth was therefore obtained.

Serial measurements of head circumference and crown-heel length should be used more often in studies of infant growth. These take very little time to perform and, provided care is taken, they are highly reproducible. The uncertainty of optimum milk requirements for low-birth-weight infants might be sooner resolved if these more meaningful measures of growth are evaluated in addition to weight gain.-I am, etc.,

Department of Child Health,

Welsh National School of Medicine,

D. P. Davies 1 Babson, S. G., and Bramhall, J. L., fournal of
Pediatrics, 1969, 74, 890.
2 Bray, P. F., et al., fournal of Pediatrics, 1969, $75,303$. .
Winick, M., and Rosso, P., Pediatric Research, 1969, 3, 181.
4 Davies, D. P., and Holding, R. E., Archives of
Disease in Childhood, 1972, 47, 938.

5 Gairdner, D., and Pearson, J., Archives of Disease in Childhood, 1971, 46, 783.,

6 Usher, R. H., and McLean, F. H., in Scientific Foundations of Paediatrics, ed. by J. A. Davis,

\section{Misleading Drug Advertising}

SIR, $\rightarrow$ It is a pity that our profession allows itself dual standards. Were Dr. L. F. Prescott's letter' (8 March, p. 572) one of com- ment on a professional colleague's scientific work he would no doubt have extended the courtesy of sending a copy of his letter to that colleague, probably before publication, in case there were a justifiable rebuttal. I am sure Dr. Prescott knows that some of his professional colleagues are employed in the pharmaceutical industry and that to a greater or lesser degree they are involved in what their companies say and how they say it. However, I did not have the opportunity of answering Dr. Prescott's viewpoints privately and must therefore beg space to do so publicly.

Sotacor (sotalol hydrochloride) is a catecholamine antagonist initially developed by the Bristol Group because of its unique pharmacological activity. It is the only drug which blocks all adrenergic beta-receptors and has no membrane-stabilizing activity or intrinsic sympathomimetic activity. ${ }^{1}$ This, we believe, makes it singularly appropriate for the treatment of hypertension. In clinical practice, unlike propranolol, sotalol does not exert a myocardial depressant effect, ${ }^{2}$ which in some patients can precipitate left ventricular failure. Also the absence of intrinsic sympathomimetic activity avoids a paradoxical increase in blood pressure sometimes seen with other beta-adrenergic blocking agents such as oxprenolol. Further points which support the "tailor-made" concept for sotalol include: (1) its exceptionally long half life (approximately 13 hours ${ }^{1}$ ) which permits twice-daily dosage-a desirable feature in the management of hypertension; and (2) its independence of plasma renin levels ${ }^{3}$ - unlike propranolol.

Though many patients with hypertension are asymptomatic, earlier therapeutic regimens have been notorious for iatrogenic symptomatology. Against this background sotalol therapy has been associated with improved well-being. ${ }^{4}$ Sotalol may not be unique in controlling blood pressure during exercise, but the lack of this feature would be undesirable and a statement of its presence is necessary.

Dr. Prescott may not be aware that investigators from many parts of the world convened to review sotalol at a symposium in May 1974. Their contributions, which have been published, provide the endorsement for sotalol from international opinion. -I am, etc.,

Slough

A. J. JOUHAR Medical Director, Bristol Laboratories

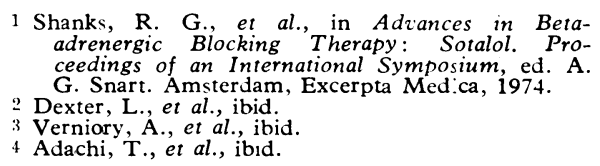

\section{Treatment of Sciatica}

SIR,-A trial, financed by $£ 30000$ from the Department of Health, of manipulation, traction, and exercises in sciatica has recently started at four London hospitals. But it is predictable that no difference will be found between these treatments, which will all give equally poor results.

According to the protocol, patients without gross bone disease or other specified contraindications are admitted to the trial merely on the basis of having pain of sciatic distribution, and they are selected for one or other of the treatments at random. Though the presence of neurological signs is noted, this is not taken into account in allocating patients to treatment groups. Now traction and manipulation constitute the main treatments for reducible disc lesions, whereas neither is of any avail for rreducible displacements. Therefore in cases of sciatica with neurological deficit both manipulation and traction are equally ineffective. ${ }^{1}$ But since the treatment groups will consist of a heterogeneous collection of patients with reducible and irreducible displacements, no valid comparisons can be made between them. In any case the fact that traction is useless in sciatica with neurological signs has been confirmed by Weber. ${ }^{2}$

To be valid a trial of sciatica treated by manipulation or traction should be carried out only on patients without signs of impaired conduction. On the other hand, if neurological signs are present epidural local anaesthesia has been shown to be a successful treatment. ${ }^{3}$

I should therefore like to suggest that, difficult though it may be, the protocol of the trial be changed to give meaningful results. - I am, etc.,

London N.W.3

GABRIEL SYMONDS

1 Cyriax, J., Texthook of Orthopaedic Medicine, vol. 1, 5th edn., pp. 520, 528. London, Bailliere, Tindall, and Cassell, 1969.

2 Weber, H., Journal of the Oslo City Hospital, 1973, 23, 167

3 Goomes, E. W., British Medical fournal, 1961, $1,20$.

\section{Overseas Doctors in the U.K}

SIR,-Dr. T. A. Reddy (1 March, p. 515) may well be right in suggesting that overseas graduates who stay in the U.K. too long become reluctant to retunn home. ${ }^{1}$ I have adduced reasons for believing that two or three years is much too long and that such reluotance becomes established in as little as 18 months. ${ }^{2}$ But we will not help foreign doctors, or ourselves, by persuading the General Medical Council to impose irritating regulations on them. We may fail to persuade such doctons to limit their stay in their own and their home countries' interests, but coercion will make our arguments less persuasive, not more. And-our interpretation of the evidence may even be wrong.-I am, etc.,

Hay on Wye, Hereford

JAMES MATHERS

1 Lancet, 1974, 1, 401. Mathers, J.

\section{Distribution of Hospital Provision}

SIR,-The paper by Mr. M. J. Buxton and Mr. R. E. Klein (8 February, p. 345) was valuable in pointing to the continuing anomalies of resource allocation within the N.H.S. It was unfortunate that only eight regions were able to provide data for the analysis. From profiles kindly provided by area medical officers I have been able to calculate comparable statistics for the five areas in the South-east Thames Region for 1972 (see table). It will be seen that the area health authority (teaching) has a striking disproportion of general medical beds, and of the A.H.A.(T.)s recorded by Mr. Buxton 